\title{
RECIL MR
}

National Cancer Institute

\section{Source}

National Cancer Institute. RECIL MR. NCI Thesaurus. Code C159620.

Greater than or equal to 10 percent decrease in the sum of longest diameters of target lesions but not a partial response. 